\title{
Adiposity and the risk of thyroid nodules with a high-suspicion sonographic pattern: a large cross-sectional epidemiological study
}

\author{
Xingjian Lai ${ }^{1}$, Bo Zhang ${ }^{2}$, Yong Wang ${ }^{3}$, Yuxin Jiang ${ }^{1}$, Jianchu Li $^{1}$, Luying Gao ${ }^{1}$, Ying Wang ${ }^{1}$ \\ ${ }^{1}$ Department of Ultrasound, Chinese Academy of Medical Sciences \& Peking Union Medical College Hospital, Beijing 100730, China; ${ }^{2}$ Department \\ of Ultrasound, China-Japan Friendship Hospital, Beijing 100029, China; ${ }^{3}$ Department of Ultrasound, National Cancer Center/National Clinical \\ Research Center for Cancer/Cancer Hospital, Chinese Academy of Medical Sciences and Peking Union Medical College, Beijing 100021, China \\ Contributions: (I) Conception and design: B Zhang, Y Wang; (II) Administrative support: Y Jiang, J Li; (III) Provision of study materials or patients: \\ Y Jiang, J Li; (IV) Collection and assembly of data: X Lai, L Gao; (V) Data analysis and interpretation: X Lai, Y Wang; (VI) Manuscript writing: All \\ authors; (VII) Final approval of manuscript: All authors. \\ Correspondence to: Yong Wang, MD. Department of Ultrasound, National Cancer Center/National Clinical Research Center for Cancer/Cancer \\ Hospital, Chinese Academy of Medical Sciences and Peking Union Medical College, No. 17 Pan-Jia-Yuan South Street, Chaoyang District, Beijing \\ 100021, China. Email: drwangyong77@163.com; Bo Zhang, MD. Department of Ultrasound, China-Japan Friendship Hospital, 2 Yinghua Dongjie, \\ Hepingli, Chao-Yang District, Beijing 100029, China. Email: zora19702006@163.com.
}

Background: To our knowledge, no previous studies have evaluated the association between obesity and the risk of thyroid nodules with a high-suspicion sonographic pattern defined by the 2015 American Thyroid Association management guidelines for adult patients with thyroid nodules and differentiated thyroid cancers. In this large cross-sectional epidemiological study, we investigated whether a positive association existed between anthropometric measurements and the risk of thyroid nodules.

Methods: In total, 10,504 individuals from two towns in northern China were enrolled between May to September 2016. The height and weight were measured and the body mass index (BMI), body fat percentage (BFP), and body surface area (BSA) were calculated. A logistic regression model was used to estimate age-, sex-, and study site-adjusted odds ratios (ORs) and confidence intervals (CIs) for each of the anthropometric measurements with respect to the risk of thyroid nodules.

Results: The final analyses included 9,096 participants, among whom 3,614 (39.7\%) had thyroid nodules and $365(4.0 \%)$ had thyroid nodules with a high-suspicion sonographic pattern. An increased risk of any thyroid nodules was associated with greater height, weight, BFP, and BSA in both men and women. Compared with the lowest quartile of BFP, the ORs for the highest quartile were 1.33 (95\% CI: 1.09-1.62) in women and 1.89 (95\% CI: 1.35-2.65) in men. An increased risk of thyroid nodules with a high-suspicion sonographic pattern was associated with greater weight, BMI, BFP, and BSA in both men and women. Compared with the lowest quartile of BFP, the ORs for the highest quartile were 2.19 (95\% CI: 1.32-3.64) in women and 3.10 (95\% CI: 1.31-7.36) in men.

Conclusions: Our results demonstrated that BFP was the most pronounced anthropometric factor associated with an increased risk of any thyroid nodules and thyroid nodules with a high-suspicion sonographic pattern.

Keywords: Adiposity; thyroid nodule; ultrasonography; body mass index (BMI); body fat percentage (BFP)

Submitted Sep 10, 2019. Accepted for publication Nov 27, 2019.

doi: $10.21037 /$ jtd.2019.11.79

View this article at: http://dx.doi.org/10.21037/jtd.2019.11.79

(c) Journal of Thoracic Disease. All rights reserved. 


\section{Introduction}

A thyroid nodule is a common thyroid disorder, and its incidence has been increasing in recent decades $(1,2)$. The clinical importance of thyroid nodules lies in the need to exclude thyroid cancer, which occurs in $7-15 \%$ of cases $(3,4)$. Generally, only nodules $>1 \mathrm{~cm}$ should be evaluated, since they have a greater potential to be clinically significant cancers, and attempts to diagnose and treat all small thyroid cancers to prevent exceedingly rare outcomes may cause more harm than good (4). Therefore, pathological confirmation of all thyroid cancers in a large epidemiological investigation of thyroid diseases is impossible (5,6). Fortunately, the 2015 American Thyroid Association management guidelines for adult patients with thyroid nodules and differentiated thyroid cancer has provided risk stratification based on a collection of sonographic features (4). These guidelines suggested that if a thyroid nodule had a high-suspicion sonographic pattern, the malignancy risk was $70-90 \%$ (4). Therefore, a thyroid nodule with a high-suspicion sonographic pattern can be a surrogate indicator for thyroid cancer in an epidemiological investigation (7).

Obesity is a known risk factor for many chronic conditions and cancers, and the recent increase in the incidence of thyroid nodules and thyroid cancers may partly be due to the epidemic of obesity, which is traditionally evaluated on the basis of the body mass index (BMI) (8-11). The association between obesity and thyroid diseases has been reported epidemiologically, but the results are not entirely consistent (8-17). To our knowledge, no previous studies have evaluated the association between obesity and the risk of thyroid nodules with a highsuspicion sonographic pattern. In this large cross-sectional epidemiological study, we investigated whether a positive association existed between anthropometric measurements and the risk of thyroid nodules.

\section{Methods}

\section{Patient and public involvement}

Randomised cluster sampling was used to in this study. Briefly, two towns in northern China (Huachuan in Heilongiiang Province and Yanjiao in Hebei Province) were selected by purposive sampling between May to September 2016. Three communities were randomly selected from each city to ensure a good representativeness. All households from each community were randomly selected and interviewed. Households were excluded if they had partial or total thyroidectomy history, or lived in these areas for less than 5 years. This led us to enroll 10,504 individuals older than 18 years. This cross-sectional epidemiological investigation of thyroid diseases was conducted in accordance with the tenets of the Declaration of Helsinki and was approved by the Peking Union Medical College Hospital ethics committee. Written informed consent was obtained from all participants before any sample or data collection.

\section{Data collection}

To collect data on demographic characteristics, dietary habits, and personal or family history of thyroid disease, a structured questionnaire was administered by trained staff during a face-to-face interview with the participants. Physical examination included height (nearest $0.5 \mathrm{~cm}$ ) and weight (measured to nearest $0.5 \mathrm{~kg}$ ) measurements. Height and weight were evaluated when the participants swore light clothes and were barefoot. The BMI was determined by dividing the weight in kilograms by the height in square meters $\left(\mathrm{kg} / \mathrm{m}^{2}\right)$. Body fat percentage (BFP) was estimated from the BMI by using the formula of Deurenberg et al.: $\mathrm{BFP}=(1.20 \times \mathrm{BMI})+(0.23 \times$ Age $)-(10.8 \times \mathrm{Sex})-5.4$, where age is in years and sex is set to 0 for women and 1 for men (18). Body surface area (BSA), an indicator of metabolic mass that is less affected by abnormal adipose mass than is the BMI, was calculated from the height and weight by using the formula of Du Bois and Du Bois: BSA = $0.007184 \times$ Weight $^{0.425} \times$ Height $^{0.725}(19)$.

\section{Imaging and image analysis}

Thyroid ultrasonography was performed in each participant after interview by experienced radiologists using a Philips HDI, HDI5000 or iU22 (Philips Medical Systems, Bothell, WA, USA) or a GE logiq e9 (GE Healthcare, Wauwatosa, WI, USA) with a 5- to $12-\mathrm{MHz}$ linear array transducer. Transverse-, longitudinal- and oblique-plane sonograms of the thyroid and cervical lymph nodes were acquired. The radiologists determined whether a participant had a thyroid nodule, which was a discrete lesion within the thyroid gland that was radiologically distinct from the surrounding thyroid parenchyma (4). If a participant had single or multiple thyroid nodules, the radiologists determined whether any thyroid nodules had a high-suspicion sonographic pattern. If a solid hypoechoic nodule or solid 
hypoechoic component of a partially cystic nodule had one or more of the following features: irregular margins (infiltrative or microlobulated), microcalcifications, taller than wide shape, rim calcifications with small extrusive soft tissue component, or evidence of extrathyroidal extension, the nodule was determined to be a thyroid nodule with a high-suspicion sonographic pattern (4).

\section{Statistical analysis}

Continuous variables were summarized as the means and ranges. Categorical variables were summarized as percentages. The chi-square test or Fisher's exact test was used to compare between different groups, as appropriate. A logistic regression model was used to estimate age-, sex-, and study site-adjusted odds ratios (ORs) and confidence intervals (CIs) for each of the anthropometric measurements with respect to the risk of any thyroid nodules and thyroid nodules with a high-suspicion sonographic pattern, including the height, weight, BFP, BSA, and BMI. In these analyses, the anthropometric measurements were examined as continuous variables and as categorical variables. For the analysis as categorical variables, the measurements were categorized into quartiles on the basis of their distribution among participants of each sex separately. Tests for trend were performed by treating ordinal category scales of the anthropometric measurements as continuous variables. Tests for interaction were determined using the Wald chi-square test of the interaction term (10). Statistical significance was determined when the $\mathrm{P}$ value was less than 0.05 . All statistical analyses were performed using SPSS for Windows/Macintosh, Version 11.5 (SPSS Inc., Chicago, IL, USA).

\section{Results}

In total, 10,504 individuals were recruited, but 1,408 were excluded from the analyses because of the absence of anthropometric measurements. The final analyses included 9,096 participants, including 2,135 (23.5\%) men and 6,961 (76.5\%) women. Of the 9,096 participants, 3,614 (39.7\%) had thyroid nodules, and 365 (4.0\%) had thyroid nodules with a high-suspicion sonographic pattern. Table 1 shows the characteristics of the participants and the distribution of anthropometric measurement among men and women.

Table 2 shows the risk analysis results of thyroid nodules associated with continuous anthropometric measurements.
The study showed a significantly increased risk of thyroid nodules per $5-\mathrm{cm}$ increase in height, per $10-\mathrm{kg}$ increase in weight, per $5 \%$ increase in BFP, per $0.5-\mathrm{m}^{2}$ increase in BSA, and per $5 \mathrm{~kg} / \mathrm{m}^{2}$ increase in the BMI (all $\left.\mathrm{P}<0.05\right)$. However, the risk of thyroid nodules in women was not significantly increased per $5 \mathrm{~kg} / \mathrm{m}^{2}$ increase in the BMI $(\mathrm{P}=0.102)$.

Table 3 shows the risk analysis results of thyroid nodules by using categorized anthropometric measurements. An association existed between height and thyroid nodule risk in men $\left(\mathrm{P}_{\text {trend }}=0.019\right)$ but not in women $\left(\mathrm{P}_{\text {trend }}=0.102\right)$. Increased weight, BFP and BSA were all significantly associated with an increased risk of thyroid nodules regardless of sex and in a dose-related manner (all $\mathrm{P}_{\text {trend }}$ $<0.05)$. The most pronounced results were for BFP, for which the OR was 1.33 in women whose BFP was $\geq 36.2 \%$ than in women whose BFP was $\leq 29.3 \%$, and the OR was 1.89 in men whose BFP was $\geq 27.7 \%$ than in men whose $\mathrm{BFP}$ was $\leq 21.6 \%$. BMI was also significantly associated with an increased risk of thyroid nodules in men $\left(\mathrm{P}_{\text {trend }}=0.004\right)$ but not in women $\left(\mathrm{P}_{\text {trend }}=0.051\right)$.

Table 4 shows the risk analysis results of thyroid nodules with a high-suspicion sonographic pattern associated with continuous anthropometric measurements. The study showed a significantly increased risk of thyroid nodule with a high-suspicion sonographic pattern per $10 \mathrm{~kg}$ increase in weight, per $5 \%$ increase in BFP, per $0.5-\mathrm{m}^{2}$ increase in $\mathrm{BSA}$, and per $5 \mathrm{~kg} / \mathrm{m}^{2}$ increase in the BMI (all $\mathrm{P}<0.05$ ). However, the risk of thyroid nodules with a high-suspicion sonographic pattern in men was not significantly increased per 5-cm increase in height $(\mathrm{P}=0.710)$.

Table 5 shows the risk analysis results of thyroid nodules with a high-suspicion sonographic pattern by using categorized anthropometric measurements. An association existed between height and the risk of thyroid nodules with a high-suspicion sonographic pattern in women $\left(\mathrm{p}_{\text {trend }}\right.$ $=0.034)$ but not in men $\left(\mathrm{P}_{\text {trend }}=0.449\right)$. Increased weight and BFP were both significantly associated with an increased risk of thyroid nodule with a high-suspicion sonographic pattern regardless of sex and in a dose-related manner (all $\mathrm{P}_{\text {trend }}<0.05$ ). The most pronounced results were for BFP, for which the OR was 2.19 in women whose BFP was $\geq 36.2 \%$ than in women whose BFP was $\leq 29.3 \%$, and the OR was 3.10 in men whose BFP was $\geq 27.7 \%$ than in men whose $\mathrm{BFP}$ was $\leq 21.6 \%$. The BMI was also significantly associated with an increased risk of thyroid nodules with a highsuspicion sonographic pattern in women $\left(\mathrm{P}_{\text {trend }}=0.003\right)$ but 
Table 1 Characteristics of participants from Huachuan and Yanjiao, China

\begin{tabular}{|c|c|c|c|c|}
\hline Characteristic & Total & No nodule & Nodules* & High-suspicion nodules ${ }^{*}$ \\
\hline Number & 4,123 & 2,580 & 1,543 & 169 \\
\hline \multicolumn{5}{|l|}{ Gender } \\
\hline Male (\%) & $839(20.3)$ & $589(22.8)$ & 250 (16.2) & $20(11.8)$ \\
\hline Age, years & $40[18-68]$ & 39 [18-68] & 42 [20-67] & 42 [22-65] \\
\hline Height, cm & 163 [140-188] & $163[140-187]$ & $162[142-188]$ & $162[150-178]$ \\
\hline Weight, kg & 64 [38-130] & 64 [38-115] & $64[40-130]$ & 65 [44-95] \\
\hline BFP, \% & $30.4(9.6-63.1)$ & $29.7(9.6-52.8)$ & $31.6(11.3-63.1)$ & $32.4(19.7-42.6)$ \\
\hline \multicolumn{5}{|l|}{ Yanjiao } \\
\hline Number & 4,973 & 2,902 & 2,071 & 195 \\
\hline Male (\%) & 1,296 (26.1) & $878(30.3)$ & $418(20.2)$ & $37(19.0)$ \\
\hline Female (\%) & 3,677 (73.9) & $2,024(69.7)$ & $1,653(79.8)$ & $158(81.0)$ \\
\hline Age, years & 48 [18-89] & $45[18-84]$ & 52 [18-89] & $52[25-77]$ \\
\hline Height, cm & 164 [143-194] & 164 [145-188] & $163[143-194]$ & 163 [147-182] \\
\hline Weight, kg & 64 [38-130] & 64 [40-130] & 64 [38-110] & 65 [44-110] \\
\hline BFP, \% & $31.2(9.4-65.7)$ & $30.0(9.5-65.7)$ & $33.0(9.4-56.6)$ & $33.8(17.2-46.2)$ \\
\hline Female (\%) & $6,961(76.5)$ & $4,015(73.2)$ & $2,946(81.5)$ & $307(84.3)$ \\
\hline Age, years & $44(18-89)$ & $42[18-84]$ & 47 [18-89] & $47[22-77]$ \\
\hline Height, cm & 163 [140-194] & 164 [140-188] & 163 [142-194] & 163 [147-182] \\
\hline Weight, kg & 64 [38-130] & 64 [38-130] & 64 [38-130] & 65 [44-110] \\
\hline BFP, \% & $30.9(9.4-65.7)$ & $29.8(9.5-65.7)$ & $32.4(9.4-63.1)$ & $33.1(17.2-46.2)$ \\
\hline $\mathrm{BSA}, \mathrm{m}^{2}$ & $1.69(1.27-2.40)$ & $1.69(1.28-2.35)$ & $1.68(1.27-2.40)$ & $1.69(1.40-2.30)$ \\
\hline BMI, kg/m² & $23.9(14.9-50.8)$ & 23.7 (15.4-48.9) & $24.1(14.9-50.8)$ & $24.5(16.5-35.2)$ \\
\hline
\end{tabular}

*, "Nodules" include "high-suspicion nodules". Data are presented as mean (minimum - maximum). BFP, body fat percentage; BSA, body surface area; BMI, body mass index. 
Table 2 Odds ratios and confidence intervals for thyroid nodule risk for continuous anthropometric measurements

\begin{tabular}{|c|c|c|}
\hline Variable & OR (95\% Cl) & $\mathrm{P}$ \\
\hline \multicolumn{3}{|c|}{ Height (5-cm increment) } \\
\hline Overall & 1.07 (1.03-1.12) & 0.001 \\
\hline Female & $1.05(1.00-1.11)$ & 0.046 \\
\hline Male & $1.11(1.03-1.21)$ & 0.011 \\
\hline \multicolumn{3}{|c|}{ Weight (10-kg increment) } \\
\hline Overall & $1.12(1.07-1.18)$ & $<0.001$ \\
\hline Female & 1.09 (1.03-1.15) & 0.003 \\
\hline Male & 1.19 (1.09-1.29) & $<0.001$ \\
\hline \multicolumn{3}{|c|}{ BFP (5\% increment) } \\
\hline Overall & $1.10(1.05-1.16)$ & $<0.001$ \\
\hline Female & $1.07(1.01-1.14)$ & 0.029 \\
\hline Male & 1.18 (1.05-1.32) & 0.004 \\
\hline \multicolumn{3}{|c|}{ BSA (0.5- $\mathrm{m}^{2}$ increment) } \\
\hline Overall & $1.17(1.06-1.30)$ & 0.002 \\
\hline Female & $1.14(1.02-1.27)$ & 0.026 \\
\hline Male & $1.35(1.02-1.78)$ & 0.037 \\
\hline \multicolumn{3}{|c|}{ BMI (5-kg/m² increment) } \\
\hline Overall & $1.10(1.04-1.17)$ & 0.002 \\
\hline Female & $1.06(0.99-1.14)$ & 0.102 \\
\hline Male & $1.20(1.06-1.38)$ & $<0.001$ \\
\hline
\end{tabular}

Odds ratios were adjusted for age, sex, and study area. OR, odds ratio; $\mathrm{Cl}$, confidence interval; BFP, body fat percentage; BSA, body surface area; BMI, body mass index.

not in men $\left(\mathrm{P}_{\text {trend }}=0.072\right)$.

\section{Discussion}

This study evaluated the associations between anthropometric measurements and the risk of thyroid nodules with a high-suspicion sonographic pattern defined by the 2015 American Thyroid Association management guidelines for adult patients with thyroid nodules and differentiated thyroid cancer. Among the anthropometric measurements examined, BFP had the strongest association with the risk of thyroid nodules with a high-suspicion sonographic pattern. Specifically, men with a BFP $\geq 27.7 \%$ had approximately three times the risk of thyroid nodules with a high-suspicion sonographic pattern in the lowest quartile of BFP, and women with a BFP $\geq 36.2 \%$ had approximately two times the risk of thyroid nodules with a high-suspicion sonographic pattern in the lowest quartile of BFP. For thyroid nodules, we obtained similar results. Among the anthropometric measurements examined, BFP had the strongest association with the risk of thyroid nodules.

Similar to our results, $\mathrm{Xu}$ et al. also found that BFP had the strongest association with the risk of papillary thyroid cancer (10). In the pooled analysis of three casecontrol populations, men with a BFP $>27.9 \%$ and women with a BFP $>37.8 \%$ had approximately four times the papillary thyroid cancer risk than did men and women in the lowest quartile of BFP (10). The results of both studies demonstrated that BFP was useful as a predictor of thyroid nodules or cancer risk, and added to the existing evidence suggesting that greater body fatness was associated with a higher thyroid cancer risk.

The BMI is a frequently used indicator of body fatness in epidemiological studies. However, in the present study, the BMI was significantly associated with an increased risk of thyroid nodules with a high-suspicion sonographic pattern in women $\left(\mathrm{p}_{\text {trend }}=0.003\right)$ but not in men $\left(\mathrm{p}_{\text {trend }}=0.072\right)$. Only 57 men had thyroid nodules with a high-suspicion sonographic pattern in this study; therefore, the nonsignificant association between the BMI and risk of thyroid nodules with a high-suspicion sonographic pattern in men was likely due to the small number of male participants (10). Interestingly, some prior studies obtained similar results. Dal Maso et al. found a significant but moderately increased risk of thyroid cancer with a higher BMI in women [relative risk for highest tertile, 1.2 (95\% CI: 1.0-1.4)] but not for men (20). Cléro et al. found a significantly increased risk of differentiated thyroid cancer with a higher $\mathrm{BMI}$ in women [OR for highest tertile, 3.0 (95\% CI: 2.0-14.48)] but not for men (21). Those two studies included only 51 and 65 male patients, respectively $(20,21)$. By contrast, the analysis conducted by $\mathrm{Xu}$ et al. included 557 male patients and demonstrated a statistically significant association between BMI and papillary thyroid cancer risk in men (10). Therefore, we should include more male patients in future studies.

In this study, we found that a greater BMI was associated with a risk of thyroid nodules with a high-suspicion sonographic pattern among men [OR per $5-\mathrm{kg} / \mathrm{m}^{2}$ increment, 1.67 (1.16-2.39)] and among women [1.20 (1.02-1.41)]. In other words, men had a slightly higher 
Table 3 Odds ratios and confidence intervals for thyroid nodule risk according to the categories of anthropometric measurements

\begin{tabular}{|c|c|c|c|c|c|c|c|c|c|}
\hline \multirow{2}{*}{ Variable } & \multicolumn{2}{|r|}{ Q1* } & \multicolumn{2}{|r|}{ Q2* } & \multicolumn{2}{|r|}{ Q3* $^{*}$} & \multicolumn{2}{|r|}{$\mathrm{Q}^{*}$} & \multirow{2}{*}{$P_{\text {trend }}$} \\
\hline & Range & OR $(95 \% \mathrm{Cl})$ & Range & OR $(95 \% \mathrm{Cl})$ & Range & OR (95\% Cl) & Range & OR $(95 \% \mathrm{Cl})$ & \\
\hline Height (cm), women & $\leq 157$ & 1.0 & $158-160$ & $1.07(0.94-1.23)$ & $161-163$ & $1.01(0.86-1.12)$ & $\geq 164$ & $1.16(1.00-1.35)$ & 0.102 \\
\hline Weight (kg), women & $\leq 54$ & 1.0 & $55-60$ & $1.04(0.91-1.19)$ & $61-65$ & $1.21(1.04-1.41)$ & $\geq 66$ & $1.16(1.00-1.35)$ & 0.016 \\
\hline Weight (kg), men & $\leq 65$ & 1.0 & $66-74$ & $1.47(1.12-1.93)$ & $75-80$ & $1.30(0.99-1.71)$ & $\geq 81$ & $1.62(1.22-2.16)$ & 0.003 \\
\hline BFP (\%), men & $\leq 21.6$ & 1.0 & $21.7-24.5$ & $1.24(0.92-1.68)$ & $24.6-27.6$ & $1.40(1.02-1.92)$ & $\geq 27.7$ & $1.89(1.35-2.65)$ & $<0.001$ \\
\hline BSA $\left(\mathrm{m}^{2}\right)$, women & $\leq 1.55$ & 1.0 & $1.56-1.62$ & $1.06(0.92-1.22)$ & $1.63-1.70$ & $1.13(0.99-1.30)$ & $\geq 1.71$ & $1.21(1.05-1.40)$ & 0.005 \\
\hline $\mathrm{BSA}\left(\mathrm{m}^{2}\right)$, men & $\leq 1.76$ & 1.0 & $1.77-1.85$ & $1.25(0.95-1.65)$ & $1.86-1.96$ & $1.32(1.01-1.74)$ & $\geq 1.96$ & $1.55(1.16-2.08)$ & 0.002 \\
\hline BMI $\left(\mathrm{kg} / \mathrm{m}^{2}\right)$, women & $\leq 21.3$ & 1.0 & 21.4-23.3 & $1.17(1.01-1.35)$ & $23.4-25.4$ & 1.15 (1.00-1.32) & $\geq 25.5$ & $1.18(1.02-1.38)$ & 0.051 \\
\hline
\end{tabular}

Odds ratios were adjusted for age, sex, and study area. ${ }^{*}$, for the analysis as categorical variables, measurements were categorized into quartiles on the basis of their distribution. Q1, Q2, Q3 and Q4 is the $1^{\text {st }}, 2^{\text {nd }}, 3^{\text {rd }}$ and $4^{\text {th }}$ quartiles, respectively. Q, quartile; OR, odds ratio; $\mathrm{Cl}$, confidence interval; BFP, body fat percentage; BSA, body surface area; BMI, body mass index.

Table 4 Odds ratios and confidence intervals for thyroid nodules with a high-suspicion sonographic pattern risk for continuous anthropometric measurements

\begin{tabular}{|c|c|c|}
\hline Variable & OR $(95 \% \mathrm{Cl})$ & $P$ \\
\hline \multicolumn{3}{|c|}{ Height (5-cm increment) } \\
\hline Overall & $1.11(1.01-1.23)$ & 0.040 \\
\hline Female & $1.13(1.01-1.27)$ & 0.036 \\
\hline Male & $1.05(0.83-1.32)$ & 0.710 \\
\hline \multicolumn{3}{|c|}{ Weight (10-kg increment) } \\
\hline Overall & $1.23(1.11-1.37)$ & $<0.001$ \\
\hline Female & $1.22(1.08-1.37)$ & 0.001 \\
\hline Male & $1.31(1.04-1.64)$ & 0.021 \\
\hline \multicolumn{3}{|c|}{ BFP (5\% increment) } \\
\hline Overall & $1.30(1.18-1.43)$ & $<0.001$ \\
\hline Female & $1.20(1.05-1.38)$ & 0.008 \\
\hline Male & $1.68(1.28-2.20)$ & $<0.001$ \\
\hline \multicolumn{3}{|c|}{ BSA $\left(0.5-\mathrm{m}^{2}\right.$ increment) } \\
\hline Overall & $1.37(1.08-1.73)$ & 0.008 \\
\hline Female & $1.29(1.01-1.65)$ & 0.041 \\
\hline Male & $2.68(1.10-6.54)$ & 0.031 \\
\hline \multicolumn{3}{|c|}{ BMI (5-kg/m² increment) } \\
\hline Overall & $1.26(1.09-1.46)$ & 0.002 \\
\hline Female & $1.20(1.02-1.41)$ & 0.025 \\
\hline Male & $1.67(1.16-2.39)$ & 0.006 \\
\hline
\end{tabular}

risk of thyroid nodules with a high-suspicion sonographic pattern as the BMI increased. A recent meta-analysis obtained similar results and found that a greater BMI was associated with a risk of thyroid cancer among men [relative risk per $5 \mathrm{~kg} / \mathrm{m}^{2}$ increment, $\left.1.41(1.11-1.78)\right]$ and among women [1.25 (1.14-1.38)] (22). These results might reflect the sex differences in the proportion of subcutaneous fat and visceral fat (16). As the BMI increased, women tended to have more subcutaneous fat than visceral fat, while men tended to have more visceral fat than subcutaneous fat $(23,24)$. Individuals with a greater proportion of visceral fat are at a higher risk of insulin resistance and metabolic syndrome than are those with more subcutaneous fat $(23,25)$. Some studies have demonstrated the association between insulin resistance and thyroid nodules. Ayturk et al. found that insulin resistance might be associated with thyroid-nodule formation more strongly than even waist circumference might be (26). Wang et al. found that insulin resistance was associated with the distribution, construction, and density of blood vessels in thyroid nodules (27). These results might also explain that BFP had the strongest association with the risk of thyroid nodules with a highsuspicion sonographic pattern, because BFP was a better indicator of body fatness, which took in account both age and sex (18).

To our knowledge, this is the first large cross-sectional epidemiological study to evaluate the association between obesity and the risk of any thyroid nodules and thyroid 
Table 5 Odds ratios and confidence intervals for thyroid nodules with a high-suspicion sonographic pattern risk according to the categories of anthropometric measurements

\begin{tabular}{|c|c|c|c|c|c|c|c|c|c|}
\hline Variable & \multicolumn{2}{|r|}{ Q1* } & \multicolumn{2}{|r|}{ Q2* } & \multicolumn{2}{|r|}{ Q3* $^{*}$} & \multicolumn{2}{|r|}{ Q4* } & $P_{\text {trend }}$ \\
\hline Height (cm), women & $\leq 157$ & 1.0 & $158-160$ & $1.22(0.88-1.69)$ & $161-163$ & $1.28(0.85-1.90)$ & $\geq 164$ & $1.50(1.06-2.12)$ & 0.034 \\
\hline Height (cm), men & $\leq 169$ & 1.0 & $170-171$ & $1.11(0.50-2.48)$ & $172-175$ & $1.51(0.75-3.02)$ & $\geq 176$ & $1.14(0.47-2.78)$ & 0.449 \\
\hline Weight (kg), women & $\leq 54$ & 1.0 & $55-60$ & $1.43(1.00-2.05)$ & $61-65$ & $1.52(1.04-2.24)$ & $\geq 66$ & $1.78(1.22-2.59)$ & 0.003 \\
\hline BFP (\%), women & $\leq 29.3$ & 1.0 & $29.4-32.7$ & $2.01(1.40-3.13)$ & $32.8-36.1$ & $1.52(0.95-2.45)$ & $\geq 36.2$ & $2.19(1.32-3.64)$ & 0.002 \\
\hline BFP (\%), men & $\leq 21.6$ & 1.0 & $21.7-24.5$ & $2.02(0.81-5.03)$ & $24.6-27.6$ & $1.05(0.37-3.00)$ & $\geq 27.7$ & $3.10(1.31-7.36)$ & $<0.001$ \\
\hline BSA $\left(\mathrm{m}^{2}\right)$, women & $\leq 1.55$ & 1.0 & $1.56-1.62$ & $1.45(1.02-2.07)$ & $1.63-1.70$ & $1.51(1.06-2.14)$ & $\geq 1.71$ & $1.74(1.23-2.46)$ & 0.002 \\
\hline $\operatorname{BSA}\left(m^{2}\right)$, men & $\leq 1.76$ & 1.0 & $1.77-1.85$ & $1.68(0.71-3.93)$ & $1.86-1.96$ & $2.15(0.98-4.72)$ & $\geq 1.96$ & $1.65(0.68-3.99)$ & 0.147 \\
\hline
\end{tabular}

Odds ratios were adjusted for age, sex, and study area. ${ }^{*}$, for the analysis as categorical variables, measurements were categorized into quartiles on the basis of their distribution. Q1, Q2, Q3 and Q4 is the $1^{\text {st }}, 2^{\text {nd }}, 3^{\text {rd }}$ and $4^{\text {th }}$ quartiles, respectively. Q, quartile; OR, odds ratio; $\mathrm{Cl}$, confidence interval; BFP, body fat percentage; BSA, body surface area; BMI, body mass index.

nodules with a high-suspicion sonographic pattern. However, this study has some limitations. First, the percentage of male participants $(23.5 \%)$ was lower in this study than in similar previous studies $(37.0-46.0 \%)(11,16)$. The reason was that the economy of the two included towns in northern China (Huachuan in Heilongjiang Province and Yanjiao in Hebei Province) was not good and most of the young man went to work in big cities. Second, although the major risk factors for thyroid nodules or cancers, including age and sex, were adjusted for in the model, other possible confounders were not assessed in our analysis, including smoking, alcohol consumption, and dietary and physical behaviors. In addition, we did not include some other anthropometric measurements which can more accurately measure central adiposity, such as waist circumference and the waist-to-hip ratio. Song et al. suggested that waist circumference was superior to the $\mathrm{BMI}$ in assessing the risk of thyroid nodules in Chinese subjects (16). However, some studies did not demonstrate that the waist-to-hip ratio was associated more strongly than the BMI was with thyroid cancer risk $(28,29)$. Therefore, the role of central adiposity in the development of thyroid nodules and cancers needs further investigation.

In conclusion, our results demonstrated that BFP was the most pronounced anthropometric factor associated with an increased risk of any thyroid nodules and thyroid nodules with a high-suspicion sonographic pattern. Therefore, BFP can be a good surrogate indicator for the BMI in future epidemiological studies on thyroid diseases.

\section{Acknowledgments}

Funding: This work is supported by Medicine and Health Innovation Project of CAMS (2018-I2M-3-003).

\section{Footnote}

Conflicts of Interest: The authors have no conflicts of interest to declare.

Ethical Statement: The authors are accountable for all aspects of the work in ensuring that questions related to the accuracy or integrity of any part of the work are appropriately investigated and resolved. This study was approved by the Peking Union Medical College Hospital ethics committee. Written informed consent was obtained from all participants before any sample or data collection.

\section{References}

1. Burman KD, Wartofsky L. Thyroid nodules. N Engl J 
Med 2016;374:1294-5.

2. Guth S, Theune U, Aberle J, et al. Very high prevalence of thyroid nodules detected by high frequency $(13 \mathrm{MHz})$ ultrasound examination. Eur J Clin Invest 2009;39:699-706.

3. Diao X, Zhan J, Chen L, et al. Quantification of solid hypo-echoic thyroid nodule enhancement with contrastenhanced ultrasound. Transl Cancer Res 2017;6:1078-87.

4. Haugen BR, Alexander EK, Bible KC, et al. 2015 American thyroid association management guidelines for adult patients with thyroid nodules and differentiated thyroid cancer: the American thyroid association guidelines task force on thyroid nodules and differentiated thyroid cancer. Thyroid 2016;26:1-133.

5. Payabvash S. Quantitative diffusion magnetic resonance imaging in head and neck tumors. Quant Imaging Med Surg 2018;8:1052-65.

6. Zhang J, Li Y, Zhao Y, et al. CT and MRI of superficial solid tumors. Quant Imaging Med Surg 2018;8:232-51.

7. Yan F, Song Z, Du M, et al. Ultrasound molecular imaging for differentiation of benign and malignant tumors in patients. Quant Imaging Med Surg 2018;8:1078-83.

8. Rinaldi S, Lise M, Clavel-Chapelon F, et al. Body size and risk of differentiated thyroid carcinomas: findings from the EPIC study. Int J Cancer 2012;131:E1004-14.

9. Wang N, Fang H, Fu C, et al. Associations of adiposity measurements with thyroid nodules in Chinese children living in iodine-sufficient areas: an observational study. BMJ Open 2017;7:e016706.

10. Xu L, Port M, Landi S, et al. Obesity and the risk of papillary thyroid cancer: a pooled analysis of three casecontrol studies. Thyroid 2014;24:966-74.

11. $\mathrm{Xu} \mathrm{W,} \mathrm{Chen} \mathrm{Z,} \mathrm{Li} \mathrm{N,} \mathrm{et} \mathrm{al.} \mathrm{Relationship} \mathrm{of} \mathrm{anthropometric}$ measurements to thyroid nodules in a Chinese population. BMJ Open 2015;5:e008452.

12. Feng S, Zhang Z, Xu S, et al. The prevalence of thyroid nodules and their association with metabolic syndrome risk factors in a moderate iodine intake area. Metab Syndr Relat Disord 2017;15:93-7.

13. Jiang H, Tian Y, Yan W, et al. The prevalence of thyroid nodules and an analysis of related lifestyle factors in Beijing communities. Int J Environ Res Public Health 2016;13:442.

14. Kim SK, Woo J, Park I, et al. Influence of body mass index and body surface area on the behavior of papillary thyroid carcinoma. Thyroid 2016;26:657-66.
15. Shin J, Kim MH, Yoon KH, et al. Relationship between metabolic syndrome and thyroid nodules in healthy Koreans. Korean J Intern Med 2016;31:98-105.

16. Song B, Zuo Z, Tan J, et al. Association of thyroid nodules with adiposity: a community-based cross-sectional study in China. BMC Endocr Disord 2018;18:3.

17. Yin J, Wang C, Shao Q, et al. Relationship between the prevalence of thyroid nodules and metabolic syndrome in the iodine-adequate area of Hangzhou, China: a cross-sectional and cohort study. Int J Endocrinol 2014;2014:675796.

18. Deurenberg P, Weststrate JA, Seidell JC. Body mass index as a measure of body fatness: ageand sex-specific prediction formulas. Br J Nutr 1991;65:105-14.

19. Du Bois D, Du Bois EF. A formula to estimate the approximate surface area if height and weight be known. 1916. Nutrition 1989;5:303-11, 312-3.

20. Dal Maso L, La Vecchia C, Franceschi S, et al. A pooled analysis of thyroid cancer studies. V. Anthropometric factors. Cancer Causes Control 2000;11:137-44.

21. Cléro E, Leux C, Brindel P, et al. Pooled analysis of two case-control studies in New Caledonia and French Polynesia of body mass index and differentiated thyroid cancer: the importance of body surface area. Thyroid 2010;20:1285-93.

22. Schmid D, Ricci C, Behrens G, et al. Adiposity and risk of thyroid cancer: a systematic review and meta-analysis. Obes Rev 2015;16:1042-54.

23. Power ML, Schulkin J. Sex differences in fat storage, fat metabolism, and the health risks from obesity: possible evolutionary origins. Br J Nutr 2008;99:931-40.

24. Lemieux S, Prud'Homme D, Bouchard C, et al. Sex differences in the relation of visceral adipose tissue accumulation to total body fatness. Am J Clin Nutr 1993;58:463-7.

25. Alberti KG, Zimmet P, Shaw J. The metabolic syndrome-a new worldwide definition. Lancet 2005;366:1059-62.

26. Ayturk S, Gursoy A, Kut A, et al. Metabolic syndrome and its components are associated with increased thyroid volume and nodule prevalence in a mild-tomoderate iodine-deficient area. Eur J Endocrinol 2009;161:599-605.

27. Wang K, Yang Y, Wu Y, et al. The association between insulin resistance and vascularization of thyroid nodules. J Clin Endocrinol Metab 2015;100:184-92.

28. Kabat GC, Kim MY, Thomson CA, et al. Anthropometric 
factors and physical activity and risk of thyroid cancer in postmenopausal women. Cancer Causes Control 2012;23:421-30.

29. Kitahara CM, Platz EA, Park Y, et al. Body fat distribution, weight change during adulthood, and thyroid cancer risk in the NIH-AARP Diet and Health Study. Int J Cancer 2012;130:1411-9.

Cite this article as: Lai X, Zhang B, Wang Y, Jiang Y, Li J, Gao L, Wang Y. Adiposity and the risk of thyroid nodules with a high-suspicion sonographic pattern: a large cross-sectional epidemiological study. J Thorac Dis 2019;11(12):5014-5022. doi: $10.21037 /$ jtd.2019.11.79 